\title{
Developing a meat supply chain network design using a multi-objective possibilistic programming approach
}

Ahmed Mohammed \& Qian Wang

\begin{abstract}
Purpose - This paper presents a study in developing a cost-effective meat supply chain network design aiming to minimizing the total cost of transportation, the number of transportation vehicles and the delivery time of meat products. The developed model was also used for determining the optimum numbers and allocations of farms and abattoirs that need to be established and the optimal quantity flow of livestock from farms to abattoirs and meat products from abattoirs to retailers.

Design/methodology/approach - A multi-objective possibilistic programming model was formulated with a focus on minimizing the total cost of transportation, the number of transportation vehicles and the delivery time of meat products. Three sets of Pareto solutions were obtained using the three different solution methods. These methods are the LP-metrics method, the $\varepsilon$-constraint method and the weighted Tchebycheff method, respectively. The TOPSIS method was used for seeking a best Pareto solution as a tradeoff decision when optimizing the three conflicting objectives.

Findings - A case study was also applied for examining the effectiveness and applicability of the developed multi-objective model and the proposed solution methods. The research concludes that the $\varepsilon$-constraint method has the superiority over the other two proposed methods as it offers a better solution outcome.

Research implications - This work addresses as interesting avenues for further research on exploring the delivery planner under different types of uncertainties and transportation means. Also, environmentalism has been becoming increasingly a significant global problem in the present century. Thus, the presented model could be extended to include the environmental aspects as an objective function.

Practical implications - The developed design methodology can be utilized for food supply chain designers. Also, it could be applied to realistic problems in the field of supply chain management.

Originality/value - The paper presents a methodology that can be used for tackle a multiobjective optimization problem of a meat supply chain network design. The proposed optimization method has the potential in solving the similar issue providing a compromising solution due to conflicting objectives in which each needs to be achieved towards an optimum outcome to survive in the competitive sector of food supply chains network.
\end{abstract}

Keywords: Meat; Supply chain design; Multi-objective optimization; Possibilistic; Management.

\section{Introduction}


A network of food supply chains covers a number of sectors involved in production, distribution and consumption of food products. For delivering a high quality of food products with minimum costs and maximum profits, different tactics can be employed (Simchi et al., 2001 \& Shankar et al., 2013); of which supply chains network design plays a key role on product quality, service levels, material flow, customer satisfaction and profitable return (Meier et al., 2012). Nevertheless, supply chain designers often encounter difficulties in making a trade-off solution due to optimization of conflicting objectives in such as minimization of costs, and maximization of profits and service levels. A good plan can also help deliver products timely from manufacturers to retailers through a supply chain network. This process involves a determination of allocations and locations of facilities, material handling capacity, transportation capability, delivery time and other performance measures.

Findings through a literature review indicate that there are a small number of publications in studying food supply chains using the multi-objective optimization approaches. Revelle \& Laporte (1996) addressed several issues in supply chains design by seeking compromised solutions known as Pareto solutions (Deb, 2001 \& Konak et al., 2006). A number of researchers applied the multi-objective optimization methods to tackle these issues. Rong et al. (2011) developed a mixed integer linear programming model for solving a production and distribution planning problem of a food supply chain. Sahar et al. (2014) proposed a multi-objective optimization model aimed at minimizing $\mathrm{CO}_{2}$ emissions of transportation of goods and the total cost in product distribution through a two-layer dairy supply chain. The similar studies were also published by Robinson and Wilcox (2008) and Pagell and $\mathrm{Wu}$ (2009). Sabri and Beamon (2000) developed a multi-objective programming model used for obtaining the optimum performance of a supply chain network considering two conflicting objectives in minimization of the total cost and maximization of volume flexibility of plants. Nozick \& Turnquist (2001) developed a mathematical model in location optimization of distribution centers considering costs of facility, inventory, transportation, and service coverage. Chan et al. (2004) presented a hybrid-genetic algorithm for solving the distribution problem of a supply chain network incorporating three objectives (i.e., costs, lead time and capacity). Chen \& Lee (2004) developed a multi-objective model of a multi-echelon supply chain network seeking a compromised solution in satisfying all the conflicting objectives including fair profit distributions, safe inventory levels, customer service levels, and uncertain demands of products. Altiparmak et al. (2006) proposed a genetic algorithm focusing on minimization of inbound and outbound distribution costs and maximization of customer services in terms of delivery time and capacity of a distribution center. Sourirajan et al. (2009) investigated a two-echelon supply chain for locating distribution centers at a minimal cost using the genetic algorithm by comparing the result using the Lagrangian heuristic approach. Paksoy et al. (2010) proposed a mixed integer linear programming model used for minimizing costs in holding and ordering goods and transportation of a supply chain. Venkatesan \& Kumanan (2012) developed a multi-objective discrete particle swarm algorithm aiming to minimize supply chain costs, lead time and maximize volume flexibility. Shankar et al. (2013) investigated a four-echelon supply chain architecture using the multi-objective evolutionary approach in order to minimize costs of facility location and shipment subject to a requirement that customer demands must be met. Niknamfar (2015) introduced a 
multi-objective non-linear model for developing a production-distribution plan in a threelevel supply chain.

Researchers attempted to tackle the randomness of a system under uncertainties using stochastic programming methods (Alonso-Ayuso et al., 2007; Listes, 2007 \& El-Sayed et al., 2010). A review of supply chain networks under uncertainties was investigated by Snyder (2006). However, the stochastic programming method has several drawbacks particularly in making strategic level decisions. Some studies indicated that the fuzzy programming method may offer a better flexibility in dealing with these issues than using the stochastic programming method (Wang \& Hsu, 2010; Qin \& Ji, 2010 \& Gholamiana et al., 2015). Petrovic et al. (1998) employed a fuzzy programming approach applied into a simulation model to acquire a compromised solution between the maximization of profit and the maximization of service level of a supply chain. Wang and Shu (2007) developed a fuzzy decision model that helps tackle the issue of uncertainties of a supply chain. Aliev et al. (2007) developed a fuzzy integrated model for solving a production-distribution problem for a supply chain network using the genetic optimization method. Zarandi et al. (2011) used the interactive fuzzy goal programming method that solves a problem in design of a closed-loop supply chain. Kannan et al. (2013) proposed an approach by ranking and selecting the best green suppliers of a supply chain according to the economic and environmental criteria. The proposed approach combines the fuzzy multi-attribute utility theory with the multi-objective programming method. Saha et al. (2015) developed a multiitem multi-objective supply chain model in a fuzzy-stochastic environment with a potential risk in estimated budgets for long-term contracts. Talaei et al. (2015) developed a biobjective model for investigating a facility location-allocation design of a closed loop supply chain network.

This paper presents a study in developing a multi-objective possibilistic model of a meat supply chain with an aim to minimizing the total transportation cost, the number of transportation vehicles and the delivery time of meat products from farms to abattoirs and from abattoirs to retailers. The research outcome shows it can be used as an aided tool to achieve a compromised solution for supply chain designers when developing a meat supply chain network in its optimal objectives.

\section{Model description and formulation}

Figure 1 illustrates a three-echelon meat supply chain network consisting of farms, abattoirs and retailers. An RFID (radio frequency identification)-based transportation system was proposed for monitoring safety and quality of meat products during the transportation process from farms to abattoirs and from abattoirs to retailers (Mohammed $\&$ Wang, 2015). RFID-based logistics and supply chains are a trend for future generation automated warehouses where customers place their orders on line and ordered goods are delivered directly to door steps of these customers (Wang et al., 2010). In order to minimize 
(1) the total transportation cost (2) the number of required vehicles for transportation (3) the delivery time. A three-objective mathematical model was developed and used for making a design decision; this also includes a determination of numbers of farms and abattoirs in response to flow of quantity of meat products between farms and abattoirs and between abattoirs and retailers.

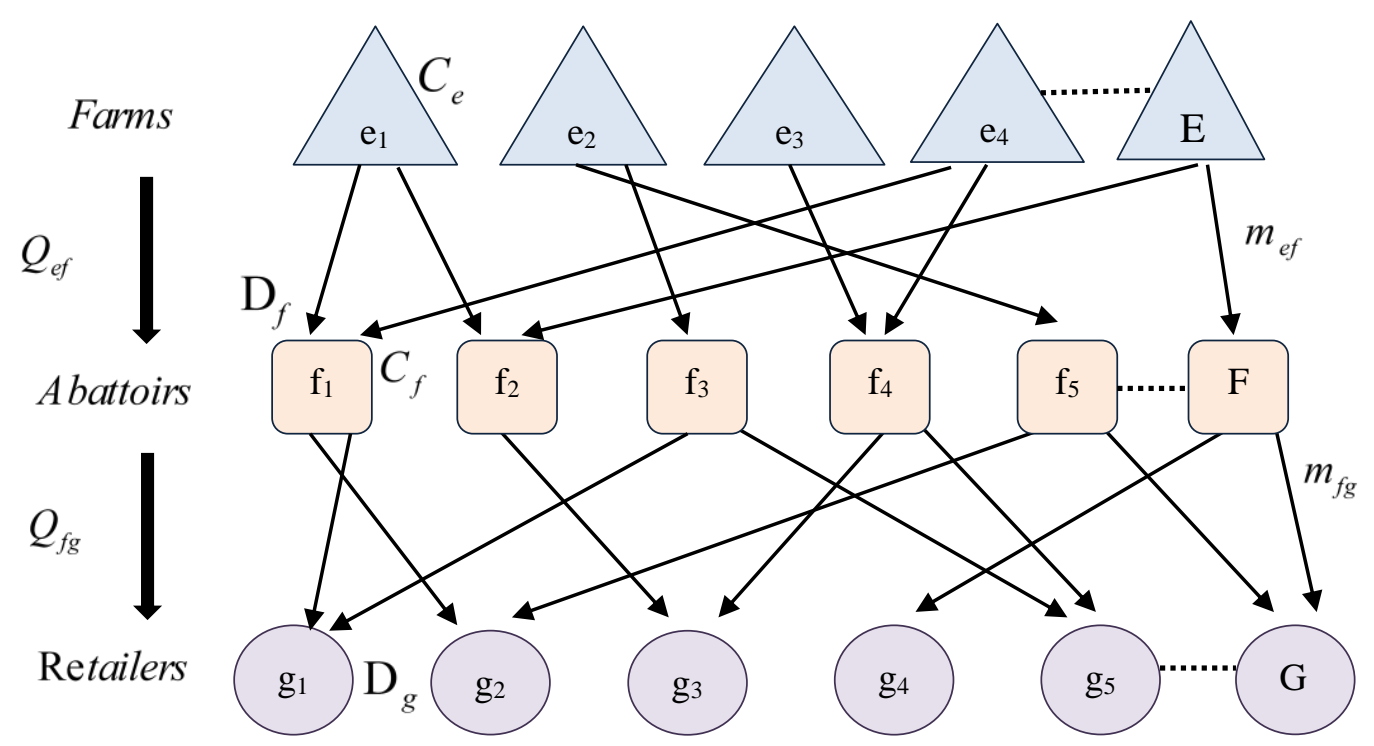

Fig. 1. The three-echelon meat supply chain network.

Notations and decision variables are described as follows:

sets

$E$ set of farms $(1 \ldots e \ldots E)$

$F$ set abattoirs $(1 \ldots \mathrm{f} \ldots \mathrm{F})$

$G$ set retailers $(1 \ldots \mathrm{g} \ldots \mathrm{G})$

\section{Parameters}

$C_{e f}^{t} \quad$ RFID tag cost (GBP) per item transported from farm $e$ to abattoir $f$

$C_{f g}^{t} \quad$ RFID tag cost (GBP) per item transported from abattoir $f$ to retailer $g$

$C_{e f}^{m / l}$ RFID reader cost (GBP) required per lorry $l$ travelling from farm $i$ to abattoir $j$

$C_{f g}^{m / l}$ RFID reader cost (GBP) required per lorry $l$ travelling from abattoir $f$ to retailer $g$

$T C_{e f}$ unit transportation cost (GBP) per mile from farm $e$ to abattoir $f$ 
$T C_{f g}$ unit transportation cost (GBP) per mile from abattoir $f$ to retailer $g$

$d_{e f}$ transportation distance (miles) of livestock from farm $e$ t abattoir $f$

$d_{f g}$ transportation distance (miles) of processed meats from abattoir $f$ to retailer $g$

$C_{l}$ transportation capacity (units) per lorry $l$

$t t_{e f}^{l}$ travel time (h) of lorry $l$ from farm $e$ to abattoir $f$

$t t_{f g}^{l}$ travel time (h) of lorry $l$ from abattoir $f$ to retailer $g$

$S^{l} \quad$ speed $(\mathrm{m} / \mathrm{h})$ of lorry $l$

$C_{e} \quad$ maximum supply capacity (units) of farm $e$

$C_{f}$ maximum supply capacity (units) of abattoir $f$

$\mathrm{D}_{f}$ minimum demand (in units) of abattoir $f$

$\mathrm{D}_{g}$ minimum demand (in units) of retailer $g$

Decision variables

$m_{e f}$ quantity of livestock transported from farm $e$ to abattoir $f$

$m_{f g}$ quantity of processed meats transported from abattoir $f$ to retailer $g$

$Q_{e f}$ number of expected required vehicles to transport livestock from farm $e$ to abattoir

$f$

$Q_{f g}$ number of expected required vehicles to transport processed meats from abattoir $f$ to retailer $g$

Binary decision variables

$u_{e}=\left\{\begin{array}{l}1: \text { if farm } e \text { is open } \\ 0: \text { otherwise }\end{array}\right.$

$v_{f}=\left\{\begin{array}{l}1: \text { if abattoir } f \text { is open } \\ 0: \text { otherwise }\end{array}\right.$

The aim of the developed three-objective model of the meat supply chain network is to minimize the total transportation cost $\mathrm{O}_{1}$, which includes (a) unit transportation cost per mile (b) RFID tag cost per unit and (c) RFID reader per vehicle, is given in Eq. 1.

$$
\begin{aligned}
& \text { Min } O_{1}=\sum_{e \in E} \sum_{f \in F} T C_{e f} m_{e f}+\sum_{f \in F} \sum_{g \in G} T C_{f g} m_{f g}+\sum_{e \in G} \sum_{f \in F} C_{e f}^{t} m_{e f}+\sum_{f \in F} \sum_{g \in G} C_{f g}^{t} m_{f g} \\
& +\sum_{e \in E} \sum_{f \in F} C_{e f}^{m / l} Q_{e f}+\sum_{f \in F} \sum_{g \in G} C_{f g}^{m / l} Q_{f g}
\end{aligned}
$$

By minimizing the number of required transportation vehicles $\mathrm{O}_{2}$, it is given in Eq. 2. 
$\operatorname{Min} O_{2}=\sum_{e \in E} \sum_{f \in F} T C_{e f} Q_{e f}+\sum_{f \in F} \sum_{g \in G} T C_{f g} Q_{f g}$

By minimizing the delivery time $\mathrm{O}_{3}$, it is given in Eq. 3 .

Min $O_{3}=\sum_{e \in E} \sum_{f \in F} t t_{e f}^{l} m_{e f}+\sum_{f \in F} \sum_{g \in G} t t_{f g}^{l} m_{f g}$

Subject to:

$$
\begin{aligned}
& \sum_{e \in E} m_{e f} \leq C_{e} \mathrm{u}_{e} \quad \forall \mathrm{f} \in F \\
& \sum_{f \in F} m_{f g} \leq C_{f} \mathrm{v}_{f} \quad \forall \mathrm{g} \in G \\
& \sum_{e \in E} \frac{d_{e f}}{S^{l}} \quad \forall f \in F \\
& \sum_{f \in F} \frac{d_{f g}}{S^{l}} \quad \forall g \in G \quad \forall \mathrm{f} \in F \\
& \sum_{e \in E} m_{e f} \geq \mathrm{D}_{f} \quad \forall \mathrm{g} \in G \\
& \sum_{f \in F} m_{f g} \geq \mathrm{D}_{g} \quad \forall \mathrm{f} \in F \\
& \mathrm{D}_{f} \geq \sum_{g \in G} m_{f g} \quad \forall f \in F \\
& \sum_{e \in E} Q_{e f}=m_{e f} / \mathrm{C}_{l} \quad \forall f \quad \forall k \in K \\
& \sum_{f \in F} Q_{f g}=m_{f g} / \mathrm{C}_{l} \quad \forall k \in K \\
& Q_{e f}, Q_{f g} \text { integer } \\
& m_{e f}, m_{f g} \geq 0 \quad \forall e, f \\
& u_{e}, v_{f} \in\{1,0\}, \quad \forall e, f
\end{aligned}
$$

Equations 4-5 are the constraints of capacity at farms and abattoirs. Equations 6-7 determine the total transportation time of meat products shipped from farms to abattoirs and from abattoirs to retailers, respectively. The total transportation time is defined as the total traveling distance of all required vehicles divided by the traveling speed of these vehicles. Equations 8-10 ensure that all the demands of abattoirs and retailers must be satisfied. Equations 11-12 give the estimated number of vehicles for objective function two. Equations 13-15 prohibit decision variables used from the non-binary and nonnegativity.

The possibilistic programming is a powerful mathematical optimization approach that can be used for tackling optimization problems under uncertainty when parameters are not clearly defined (i.e., fuzzy parameters), or an exact value is not critical to the problem. 
Thus, the multi-objective model as described above was transformed further into an equivalent crisp model using the possibilistic programming proposed by Jiménez et al. 2007 as follows:

To minimize the total transportation cost $\mathrm{O}_{1}$, it is given:

$$
\begin{aligned}
& \text { Min } O_{1}=\sum_{e \in E} \sum_{f \in F} T C_{e f} m_{e f}+\sum_{f \in F} \sum_{g \in G} T C_{f g} m_{f g}+\sum_{e \in E} \sum_{f \in F} C_{e f}^{t} u_{e}+\sum_{f \in F} \sum_{g \in G} C_{f g}^{t} v_{f} \\
& +\sum_{e \in E} \sum_{f \in F} C_{e f}^{m} Q_{e f}+\sum_{f \in F} \sum_{g \in G} C_{f g}^{m} Q_{f g}
\end{aligned}
$$

To minimize the number of transportation vehicles $\mathrm{O}_{2}$, it is given:

$$
\operatorname{Min} O_{2}=\sum_{e \in E} \sum_{f \in F}\left(\frac{Q_{e f 1}+Q_{e f 2}+Q_{e f 3}+Q_{e f 4}}{4} \cdot \mathrm{TC}_{e f}\right)+\sum_{f \in F} \sum_{g \in G}\left(\frac{Q_{f g 1}+Q_{f g 2}+Q_{f g 3}+Q_{f g} 4}{4} \cdot T C_{f g}\right)
$$

To minimize the delivery time $\mathrm{O}_{3}$, it is given:

$$
\operatorname{Min} O_{3}=\sum_{e \in E} \sum_{f \in F} t t_{e f}^{l} m_{e f}+\sum_{f \in F} \sum_{g \in G} t t_{f g}^{l} m_{f g}
$$

Subject to:

$$
\begin{array}{ll}
\sum_{e \in E} m_{e f} \leq C_{e} \mathrm{u}_{e}, \quad \forall \mathrm{f} \in F & \\
\sum_{f \in F} m_{e f} \leq C_{f} \mathrm{v}_{f}, \quad \forall \mathrm{g} \in G & \\
\sum_{e \in E} \frac{d_{e f}}{S^{l}} \quad \forall f \in F & \\
\sum_{f \in F} \frac{d_{f g}}{S^{l}} \quad \forall g \in G & \\
\sum_{e \in E} m_{e f} \geq\left[\frac{\alpha}{2} \cdot \frac{\mathrm{D}_{f 1}+\mathrm{D}_{f 2}}{2}+\left(1-\frac{\alpha}{2}\right) \frac{\mathrm{D}_{f 3}+\mathrm{D}_{f 4}}{2}\right], & \forall \mathrm{f} \in F \\
\sum_{f \in F} m_{f g} \geq\left[\frac{\alpha}{2} \cdot \frac{\mathrm{D}_{g 1}+\mathrm{D}_{g 2}}{2}+\left(1-\frac{\alpha}{2}\right) \frac{\mathrm{D}_{g 3}+\mathrm{D}_{g 4}}{2}\right], & \forall \mathrm{g} \in G \\
{\left[\frac{\alpha}{2} \cdot \frac{\mathrm{D}_{f 1}+\mathrm{D}_{f 2}}{2}+\left(1-\frac{\alpha}{2}\right) \frac{\mathrm{D}_{f 3}+\mathrm{D}_{f 4}}{2}\right] \geq \sum_{g \in G} m_{f g},} & \forall \mathrm{f} \in F \\
\sum_{e \in E}\left[\frac{\alpha}{2} \cdot \frac{Q_{e f 1}+Q_{e f 2}}{2}+\left(1-\frac{\alpha}{2}\right) \frac{Q_{e f 3}+Q_{e f 4}}{2}\right]=m_{e f} / \mathrm{C}_{l}, & \forall f \in F
\end{array}
$$




$$
\begin{aligned}
& \sum_{f \in F}\left[\frac{\alpha}{2} \cdot \frac{Q_{f g 1}+Q_{f g}}{2}+\left(1-\frac{\alpha}{2}\right) \frac{Q_{f g 3}+Q_{f g} 4}{2}\right]=m_{f g} / \mathrm{C}, \quad \forall k \in K \\
& Q_{e f}, Q_{f g} \text { integer } \\
& m_{e f}, m_{f g} \geq 0 \quad \forall e, f \\
& u_{e}, v_{f}, \alpha \in\{1,0\}, \forall e, f
\end{aligned}
$$

Knowing that constraints with uncertain parameters must be formed at least with a satisfaction level of $\alpha$.

\section{Optimization methodology}

In order to obtain the Pareto-optimal solutions, the following steps were carried out:

(1) Find the upper and lower bound $(U, L)$ solution for each objective function. This can be obtained by:

The upper bound solution is:

$$
\begin{aligned}
& \operatorname{Max} O_{1}\left(U_{1}\right)=\sum_{e \in E} \sum_{f \in F} T C_{e f} m_{e f}+\sum_{f \in F} \sum_{g \in G} T C_{f g} m_{f g}+\sum_{e \in E} \sum_{f \in F} C_{e f}^{t} u_{e} \\
& +\sum_{f \in F} \sum_{g \in G} C_{f g}^{t} v_{f}+\sum_{e \in E} \sum_{f \in F} C_{e f}^{m} Q_{e f}+\sum_{f \in F} \sum_{g \in G} C_{f g}^{m} Q_{f g} \\
& \operatorname{Max} O_{2}\left(U_{2}\right)=\sum_{e \in E} \sum_{f \in F} T C_{e f} Q_{e f}+\sum_{f \in F} \sum_{g \in G} T C_{f g} Q_{f g} \\
& \operatorname{Max}_{3}\left(U_{3}\right)=\sum_{e \in E} \sum_{f \in F} t t_{e f}^{l} m_{e f}+\sum_{f \in F} \sum_{g \in G} t t_{f g}^{l} m_{f g}
\end{aligned}
$$

The lower bound solution is:

$$
\begin{aligned}
& \operatorname{Min} O_{1}\left(L_{1}\right)=\sum_{e \in E} \sum_{f \in F} T C_{e f} m_{e f}+\sum_{f \in F} \sum_{g \in G} T C_{f g} m_{f g}+\sum_{e \in E} \sum_{f \in F} C_{e f}^{t} u_{e} \\
& +\sum_{f \in F} \sum_{g \in G} C_{f g}^{t} v_{f}+\sum_{e \in E} \sum_{f \in F} C_{e f}^{m} Q_{e f}+\sum_{f \in F} \sum_{g \in G} C_{f g}^{m} Q_{f g} \\
& \operatorname{Min} O_{2}\left(L_{2}\right)=\sum_{e \in E} \sum_{f \in F} T C_{e f} Q_{e f}+\sum_{f \in F} \sum_{g \in G} T C_{f g} Q_{f g} \\
& \operatorname{Min} O_{3}=\sum_{e \in E} \sum_{f \in F} t t_{e f}^{l} m_{e f}+\sum_{f \in F} \sum_{g \in G} t t_{f g}^{l} m_{f g}
\end{aligned}
$$


(2) Find the respective satisfaction degree $\mu\left(x_{i}\right)$ for each objective function, this can be obtained by:

$$
\begin{aligned}
& \mu_{1}\left(O_{1}(x)\right)= \begin{cases}1 & \text { if } O_{1}(x) \geq U_{1} \\
\frac{O_{1}(x)-L_{1}}{U_{1}-L_{1}} & \text { if } L_{1} \leq O_{1}(x) \leq U_{1} \\
0 & \text { if } O_{1}(x) \leq L_{1}\end{cases} \\
& \mu_{2}\left(O_{2}(x)\right)= \begin{cases}\frac{1}{\frac{O_{2}(x)-L_{2}}{U_{2}-L_{2}}} & \text { if } L_{2} \leq O_{2}(x) \leq U_{2} \\
0 & \text { if } O_{2}(x) \leq L_{2}\end{cases} \\
& \mu_{3}\left(O_{3}(x)\right)= \begin{cases}\frac{1}{\frac{O_{3}(x)-L_{3}}{U_{3}-L_{3}}} & \text { if } L_{3} \leq O_{3}(x) \leq U_{3} \\
0 & \text { if } O_{3}(x) \leq L_{3}\end{cases}
\end{aligned}
$$

(3) Transform the crisp model obtained from section 2.2 to a single objective function using the three proposed solution methods in Eq. 39, 41 and 46.

(4) Select the best Pareto-optimal solution from the three Pareto sets using the TOPSIS method.

\subsection{Solution method}

In this work, three sets of Pareto-optimal solutions were obtained using the three solution methods, which are the LP-metrics method, the $\varepsilon$-constraint method and the weighted Tchebycheff method.

\subsubsection{The LP-metrics method}

The LP-metrics method is described as follows:

1. Based on the developed multi-objective model, each of the three objectives is optimized individually to obtain the optimal objective values $O_{1}^{*}, O_{2}^{*}$ and $O_{3}^{*}$, respectively.

2. Convert the three-objective model into a modular-objective function using the following function. 


$$
\operatorname{Min} O=\left[w_{1} \frac{O_{1}-O_{1}^{*}}{O_{1}^{*}}+w_{2} \frac{O_{2}-O_{2}^{*}}{O_{2}^{*}}+w_{3} \frac{O_{3}-O_{3}^{*}}{O_{3}^{*}}\right]
$$

Subject to Eq. 19-30.

3. Determine the importance of objectives based on decision makers' preferences and the weight formula for the three objective functions is given as follows:

$$
\sum_{x=1}^{3} w_{x}, \quad \mathrm{w}_{x} \geq 0 \quad(x=1,2,3)
$$

\subsubsection{The $\varepsilon$-constraint method}

With the $\varepsilon$-constraint method, the multi-objective model can be transformed into a singleobjective model under constraints. In this case, the higher priority of the objective is given to the total transportation cost, and other objectives, which are the number of transportation vehicle and the delivery time, are treated as the $\varepsilon$-based constraints (Ehrgott, 2005; Chankong \& Haimes, 1983). The equivalent solution formula $\mathrm{O}$ can be minimized as follow:

$$
\begin{aligned}
& \text { Min } O=\sum_{e \in E} \sum_{f \in F} T C_{e f} m_{e f}+\sum_{f \in F} \sum_{g \in G} T C_{f g} m_{f g}+\sum_{e \in E} \sum_{f \in F} C_{e f}^{t} u_{e}+\sum_{f \in F} \sum_{g \in G} C_{f g}^{t} v_{f} \\
& +\sum_{e \in E} \sum_{f \in F} C_{e f}^{m} Q_{e f}+\sum_{f \in F} \sum_{g \in G} C_{f g}^{m} Q_{f g}
\end{aligned}
$$

Subject to:

$$
\begin{aligned}
& \sum_{e \in E} \sum_{f \in F}\left(\frac{Q_{e f 1}+Q_{e f 2}+Q_{e f 3}+Q_{e f 4}}{4} \cdot \mathrm{TC}_{e f}\right)+\sum_{f \in F} \sum_{g \in G}\left(\frac{Q_{f g 1}+Q_{f g 2}+Q_{f g 3}+Q_{f g} 4}{4} \cdot T C_{f g}\right) \leq \varepsilon_{1} \\
& {\left[\sum_{e \in E} \sum_{f \in F}\left(\frac{Q_{e f 1}+Q_{e f 2}+Q_{e f 3}+Q_{e f 4}}{4} \cdot \mathrm{TC}_{e f}\right)\right]^{\min } \leq\left[\sum_{e \in E} \sum_{f \in F}\left(\frac{Q_{e f 1}+Q_{e f 2}+Q_{e f 3}+Q_{e f 4}}{4} \cdot \mathrm{TC}_{e f}\right)\right]^{\max }} \\
& \left.+\sum_{f \in F} \sum_{g \in G}\left(\frac{Q_{f g 1}+Q_{f g 2}+Q_{f g 3}+Q_{f g 4}}{4} \cdot T C_{f g}\right)\right] \\
& \left.\sum_{e \in E} \sum_{f \in F} t t_{e f}^{l} m_{e f}+\sum_{f \in F} \sum_{g \in G} t t_{f g}^{l} m_{f g} \leq \varepsilon_{2}\left(\frac{Q_{f g 1}+Q_{f g 2}+Q_{f g 3}+Q_{f g} 4}{4} \cdot T C_{f g}\right)\right] \\
& {\left[\sum_{e \in E} \sum_{f \in F} t t_{e f}^{l} m_{e f}+\sum_{f \in F} \sum_{g \in G} t t_{f g}^{l} m_{f g}\right]^{\min } \leq \varepsilon_{2} \leq\left[\sum_{e \in E} \sum_{f \in F} t t t_{e f}^{l} m_{e f}+\sum_{f \in F} \sum_{g \in G} t t t_{f g}^{l} m_{f g}\right]^{\max }}
\end{aligned}
$$

And Eq. 19-30. 
In this research, objective one is optimized in Eq.41 and objective two and three are constraints in Eq.42 and 44 respectively. An increase to the $\varepsilon$ value in Eq.43 and 45 yields a Pareto set of solutions.

\subsubsection{The weighted Tchebycheff method}

With this approach the multi-objective model can be transformed into a single-objective model $O$. The purpose of the single-objective model is to minimize the distance between the ideal objective vector $O^{*}$ and the feasible objective surface (Miettinen, 1998). The solution approach function $O$ can be formulated as follows:

$$
\operatorname{Min} O=\left(\sum_{i=1}^{3} l_{i}\left|O_{i}-O_{i}^{*}\right|^{p}\right)^{\frac{1}{p}}
$$

Subject to Eq. 19-30. It is noticed, the values of objective functions vary depending on the value of $p$. Usually, $p$ is set as 1 or 2. But, other values of $p$ can also be used. In this case study, $p$ was set as 1 .

\subsubsection{The TOPSIS method}

After revealing the Pareto solutions, a final trade-off solution needs to be determined. At present, a number of approaches can be utilized to determine the best solution based on the obtained Pareto solutions. This can be achieved based on preferences of decision makers, using a decision maker or an optimization algorithm. In this work, the TOPSIS method was employed. This approach can be used for selecting a solution nearest to the ideal solution, but also the farthest from the negative ideal solution (Ramesh et al., 2012). Assuming that $\mathrm{PR}-\left\{\mathrm{PR}_{\mathrm{op}} \mid \mathrm{o}=1,2, \ldots, \mathrm{x}\right.$ (number of pareto solutions); $\mathrm{p}=1,2, \ldots, \mathrm{y}$ (number of objectives) $\}$ refers the $x * y$ decision matrix, where $P R$ is a performance rating of one of alternative Pareto solutions with respect to values of objective function. Thus, the normalized selection procedure can be formulated as follows:

$$
N P R=\frac{P R_{o p}}{\sum_{p=1}^{o} P R_{a p}}
$$

The amount of decision information can be measured by the entropy value as:

$$
E_{p}=\frac{-1}{\ln \mathrm{x}} \sum_{o=1}^{x} P R_{o p} \ln \left(P R_{o p}\right)
$$

The degree of divergence $\mathrm{D}_{\mathrm{p}}$ of the average intrinsic information contained for $p=1,2,3$, 4 can be calculated as: 


$$
D_{p}=1-E_{p}
$$

The weight value for each objective function is given by:

$$
w_{p}=\frac{D_{p}}{\sum_{k=1}^{y} D_{k}}
$$

Thus, the normalized value of the weighted objective is given by:

$$
v_{o p}=w_{o} P R_{o p}
$$

A distance between alternative solutions can be measured by the n-dimensional Euclidean distance. Thus, the distance of each alternative from the positive and negative ideal solutions is given as:

$$
\begin{aligned}
& D_{p}^{+}=\sqrt{\left\{\sum_{o=1}^{y}\left(v_{p o}-v_{o}^{+}\right)^{2}\right\}}, \quad p=1,2, \ldots, x \\
& D_{p}^{-}=\sqrt{\left\{\sum_{o=1}^{y}\left(v_{p o}-v_{o}^{-}\right)^{2}\right\}}, \quad p=1,2, \ldots, x
\end{aligned}
$$

The relative closeness of (values of) alternative solutions to (the value of) the ideal solution is expressed as follows:

$$
r c_{p}=\frac{D_{p}^{-}}{D_{p}^{+}+D_{p}^{-}}, \quad p=1,2, \ldots, x
$$

Where $D_{p}^{-} \geq 0$ and $D_{p}^{+} \geq 0$, then, clearly, $r c_{p} \in[1,0]$

The trade-off solution can be selected with the maximum $r c_{p}$ or listed in descending order based on $r c_{p}$.

\section{Case study}

In this section, a case study was used for examining the applicability of the developed mathematical model with the effectiveness of the proposed solution methods. Data was collected from the Meat Committee (HMC, 2010). A range of application data is presented in Table 1. The transportation distances between meat supply chain facilities were estimated based on Google-map. The computational results were conducted using LINGO $^{11}$ on a Corei5 2.5-gigahertz personal laptop with an RAM of 4gigbytes. 
Table 1. Collected data of the three-echelon meat supply chain.

\begin{tabular}{llll}
\hline$E=4$ & $T C_{f g}=15-20$ & $\mathrm{D}_{\mathrm{f}}=600-1.5 \mathrm{~K}$ & $C_{e f}^{t}=0.15$ \\
$F=4$ & $C_{e}=1.2 \mathrm{~K}-2.5 \mathrm{~K}$ & $\mathrm{D}_{g}=100-200$ & $C_{f g}^{t}=0.15$ \\
$G=11$ & $C_{f}=1 \mathrm{~K}-1.8 \mathrm{~K}$ & $\mathrm{P}_{e}=0.90-0.98$ & $C_{e f}^{m / l}=800$ \\
$T C_{e f}=15-20$ & $C_{l}=20-30$ & $\mathrm{P}_{f}=0.85-1$ & $C_{f g}^{m / l}=800$ \\
$d_{e f}=23-410$ & $d_{f g}=110-174$ & $S^{l}=80$ & \\
\hline
\end{tabular}

Figure 2 illustrates the locations of candidate facility in the considered region (Yorkshire/UK) which includes four farms, four abattoirs and eleven retailers.

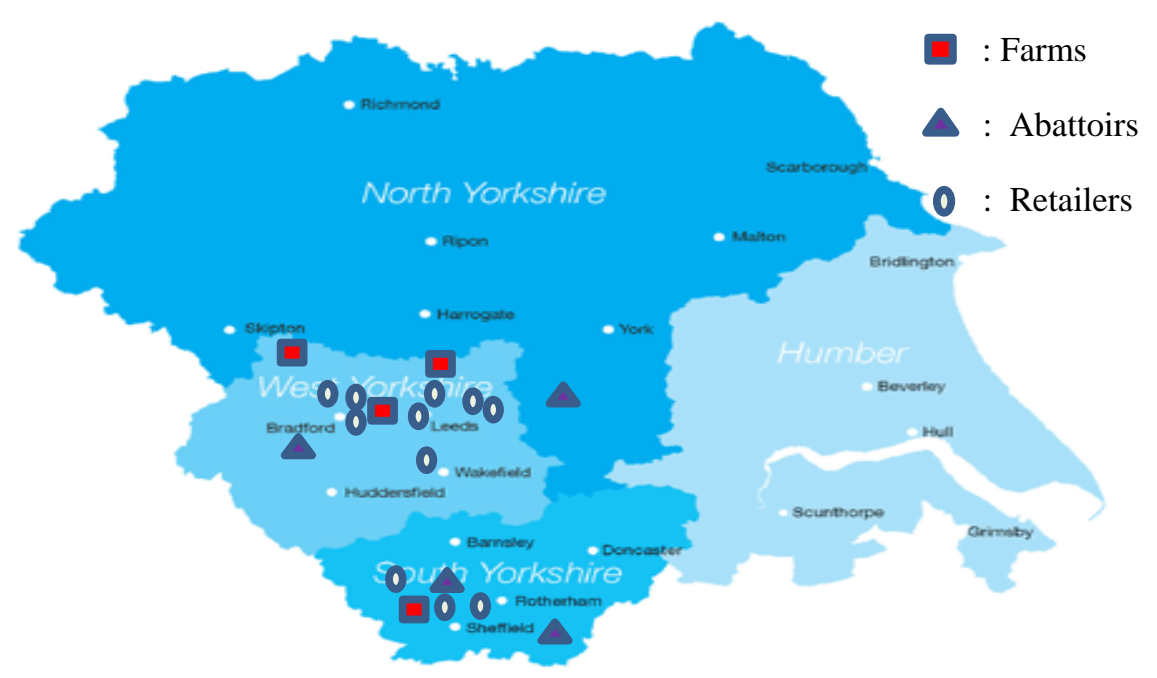

Fig. 2. Locations of candidate facilities in Yorkshire of the UK.

4.1 Results and discussions

The computational results were obtained based on the developed three-objective programming model using the three solution methods as described in section 3. Eq. 30-35 were used individually to obtain the lower value and the upper value of each objective function. The results are $\left(\left\{L_{O_{i}}, U_{O_{i}}\right\}\right)=(\{55,430,283,260\},\{26,52\},\{56,260\})$. Table 2 shows an example of the ideal values (bold values) obtained individually of each objective function. It shows the lower value and the upper value obtained based on each objective function in Eq. 30-35 individually. Table 3 shows the results of satisfaction degree $\mu\left(x_{i}\right)$ based on each objective function, shown in Eq. 36-38. 
Table 2. Optimum values obtained individually by optimizing $O i$ based on each-objective function.

\begin{tabular}{llll}
\hline Objective functions & $\min O_{1}$ & $\min O_{2}$ & $\min O_{3}$ \\
\hline$O F_{1}$ & $\mathbf{5 5 4 3 0}$ & 269360 & 187673 \\
$O F_{2}$ & 34 & $\mathbf{2 6}$ & 52 \\
$O F_{3}$ & 165 & 256 & $\mathbf{5 6}$ \\
\hline
\end{tabular}

Table 3. Result of satisfaction degree of each objective function.

\begin{tabular}{lllllllllll}
\hline$\mu\left(x_{1}\right)$ & 0.988 & 0.805 & 0.681 & 0.786 & 0.536 & 0.476 & 0.315 & 0.281 & 0.211 & 0.116 \\
$\mu\left(x_{2}\right)$ & 0.988 & 0.805 & 0.690 & 0.797 & 0.541 & 0.479 & 0.321 & 0.298 & 0.224 & 0.147 \\
$\mu\left(x_{3}\right)$ & 0.988 & 0.792 & 0.621 & 0.761 & 0.519 & 0.422 & 0.295 & 0.244 & 0.270 & 0.180 \\
\hline
\end{tabular}

The Pareto solutions are determined based on (i) the LP-metrics method; (ii) the $\varepsilon$ constraint method. Ten epsilon values were assigned from 26 to 52 of the objective function two using Eq.43, and from 56 to 260 of the objective function three using Eq.45, respectively; and (iii) the weighted Tchebycheff method (shown in Eq.46). Table 4 shows an assignment of objective-weight values used for obtaining the Pareto-optimal solutions using the LP-metrics method and the weighted Tchebycheff method. The bold values of the three objective functions, which are shown in Table 2, were given as ideal values ( $O_{1}^{*}, O_{2}^{*}, O_{3}^{*}$ ) for the solution function $O$ using Eq. 39 and 46.

Table 4. Assignment of weight values for obtaining Pareto solutions using the LP-metrics method and the weighted Tchebycheff method, respectively.

\begin{tabular}{llll}
\hline$\#$ & \multicolumn{3}{l}{ Objective weights } \\
\cline { 2 - 4 } & $w_{1}, l_{1}$ & $w_{2}, l_{2}$ & $w_{3}, l_{3}$ \\
\hline 1 & 1 & 0 & 0 \\
2 & 0.9 & 0.05 & 0.05 \\
3 & 0.8 & 0.1 & 0.1 \\
4 & 0.7 & 0.15 & 0.15 \\
5 & 0.6 & 0.2 & 0.2 \\
6 & 0.5 & 0.25 & 0.25 \\
7 & 0.4 & 0.3 & 0.3 \\
8 & 0.3 & 0.35 & 0.35 \\
9 & 0.5 & 0.3 & 0.2 \\
10 & 0.3 & 0.2 & 0.5 \\
\hline
\end{tabular}

Table 5 shows a list of the ranking Pareto-optimal solutions based on their scores using the TOPSIS method. 
Table 5. Pareto-optimal solutions ranked based on scores using the TOPSIS method.

\begin{tabular}{|c|c|c|c|}
\hline Solution & Score & & \\
\hline & LP-metrics & $\varepsilon$-constraint & Weighted Tchebycheff \\
\hline 1 & 0.245 & 0.245 & 0.245 \\
\hline 2 & 0.234 & 0.234 & 0.234 \\
\hline 3 & 0.266 & 0.266 & 0.264 \\
\hline 4 & 0.278 & 0.279 & 0.273 \\
\hline 5 & 0.253 & 0.256 & 0.256 \\
\hline 6 & 0.245 & 0.245 & 0.245 \\
\hline 7 & 0.236 & 0.234 & 0.235 \\
\hline 8 & 0.233 & 0.235 & 0.233 \\
\hline 9 & 0.231 & 0.232 & 0.233 \\
\hline 10 & 0.230 & 0.229 & 0.231 \\
\hline
\end{tabular}

Table 6 shows three sets of ten Pareto solutions obtained using the three methods, respectively as described above by assigning ten values of the satisfaction level $\alpha$ between 0.1 and 1. It also shows the optimum number of farms and abattoirs that should be established for the meat supply chain network. For instance, solution 2 is obtained based on the LP-metrics method by assigning $w_{1}=0.9, w_{2}=0.05$ and $w_{3}=0.05$. Accordingly, it gives the minimum total transportation cost of 55,430 GBP, the minimum number of required transportation vehicles of 27 and the minimum travel time of $56.4 \mathrm{~h}$. With this solution, the meat supply chain network consists of farms one and four $\left(\begin{array}{llll}1 & 0 & 0 & 1\end{array}\right)$ and abattoirs two and four ( $\left(\begin{array}{llll}0 & 1 & 0 & 1\end{array}\right)$.

Table 6. The computational results obtained by assigning the varying $\alpha$ values.

\begin{tabular}{|c|c|c|c|c|c|c|c|c|}
\hline Solution method & \# & $\begin{array}{c}\alpha- \\
\text { level }\end{array}$ & $\begin{array}{c}\operatorname{Min}\left(O_{1}\right) \\
(\mathrm{GBP})\end{array}$ & $\begin{array}{c}\operatorname{Min}\left(\mathrm{O}_{2}\right) \\
\quad \text { (unit) }\end{array}$ & $\begin{array}{c}\text { Min } \\
\left(\mathrm{O}_{3}\right)(\mathrm{h}) \\
\end{array}$ & $\begin{array}{l}\text { Open } \\
\text { farms }\end{array}$ & $\begin{array}{c}\text { Open } \\
\text { abattoirs }\end{array}$ & $\begin{array}{c}\text { Run } \\
\text { time (s) }\end{array}$ \\
\hline \multirow[t]{10}{*}{ LP-metrics } & 1 & 0.1 & 55430 & 27 & 56.4 & 10001 & 00101 & 3 \\
\hline & 2 & 0.2 & 55430 & 27 & 56.4 & 1001 & 0101 & 3 \\
\hline & 3 & 0.3 & 59343 & 29 & 78.5 & 1011 & 0101 & 2 \\
\hline & 4 & 0.4 & 64569 & 32 & 101 & 0011 & 0101 & 2 \\
\hline & 5 & 0.5 & 91234 & 34 & 123.5 & 1011 & 1011 & 4 \\
\hline & 6 & 0.6 & 224653 & 45 & 174.7 & 1111 & 1101 & 3 \\
\hline & 7 & 0.7 & 233450 & 47 & 196.1 & 1111 & 1011 & 4 \\
\hline & 8 & 0.8 & 254000 & 48 & 219.6 & 1111 & 01110 & 4 \\
\hline & 9 & 0.9 & 269360 & 50 & 239.1 & 1111 & 1011 & 4 \\
\hline & 10 & 1 & 281060 & 51 & 258.5 & 1111 & 1011 & 5 \\
\hline \multirow[t]{8}{*}{$\varepsilon$-constraint } & 1 & 0.1 & 55430 & 27 & 56.4 & 1001 & 0101 & 2 \\
\hline & 2 & 0.2 & 55430 & 27 & 56.4 & 1100 & 0110 & 2 \\
\hline & 3 & 0.3 & 59155 & 29 & 78.2 & 1101 & 0101 & 2 \\
\hline & 4 & 0.4 & 63943 & 31 & 97.5 & 1011 & 0101 & 1 \\
\hline & 5 & 0.5 & 91858 & 34 & 123.5 & 1001 & 1011 & 3 \\
\hline & 6 & 0.6 & 221340 & 44 & 168.1 & 1111 & 1101 & 4 \\
\hline & 7 & 0.7 & 233130 & 47 & 196.1 & 1111 & 1101 & 4 \\
\hline & 8 & 0.8 & 253800 & 48 & 219.6 & 1111 & 0110 & 4 \\
\hline
\end{tabular}




\begin{tabular}{|c|c|c|c|c|c|c|c|c|}
\hline & 9 & 0.9 & 269312 & 50 & 239.1 & 1111 & 1011 & 4 \\
\hline & 10 & 1 & 280950 & 50 & 255.6 & 1111 & 1011 & 3 \\
\hline Weighted & 1 & 0.1 & 55430 & 27 & 56.4 & 1001 & $\begin{array}{llll}0 & 1 & 0 & 1\end{array}$ & 3 \\
\hline & 2 & 0.2 & 55454 & 27 & 56.6 & 1001 & $\begin{array}{llll}0 & 1 & 0 & 1\end{array}$ & 3 \\
\hline & 3 & 0.3 & 59388 & 29 & 79.1 & 1011 & 1001 & 3 \\
\hline & 4 & 0.4 & 64834 & 33 & 102.6 & 1011 & 1011 & 2 \\
\hline & 5 & 0.5 & 91263 & 34 & 123.5 & 1101 & 01111 & 4 \\
\hline & 6 & 0.6 & 224653 & 45 & 175.1 & 1111 & 1101 & 4 \\
\hline & 7 & 0.7 & 233891 & 47 & 196.6 & 1111 & 1111 & 5 \\
\hline & 8 & 0.8 & 254020 & 48 & 219.6 & 1101 & $\begin{array}{llll}0 & 1 & 0 & 1\end{array}$ & 5 \\
\hline & 9 & 0.9 & 269360 & 50 & 239.2 & 1111 & 1011 & 4 \\
\hline & 10 & 1 & 283260 & 52 & 259.6 & 1111 & 1011 & 4 \\
\hline
\end{tabular}

Shown in Table 6, by increasing the satisfaction level $\alpha$, it leads to an increase of the undesired value of the three objectives. Decision makers can alter the importance of the weight value ( $w_{i}$ or $l_{i}$ ) of the three objective functions and the satisfaction level $\alpha$ based on their preferences to obtain a compromising solution as it is impossible to obtain an optimal value of all the conflicting objectives at a time. In other words, it is hard to obtain the Pareto-optimal solutions by optimizing one objective without worsening its performance in other objectives. Decision makers can also use the TOPSIS method to gain a best solution among the Pareto-optimal solutions. As shown in Table 5, with the $\varepsilon$-constraint method, solution 4 is the best solution based on its score 0.279 which is the highest. This solution was determined by assigning $\varepsilon_{1}=32$ and $\varepsilon_{2}=116.5$ that yields a minimum total transportation cost of $63,943 \mathrm{GBP}$ and a minimum travel time of $97.5 \mathrm{~h}$ with 31 transportation vehicles. The solution was also obtained based on an establishment of three farms which supplies livestock to two abattoirs. Table 7 shows the computational result of the Pareto solutions in terms of an optimum quantity of product flow between farms $(1,3$, and 4) and abattoirs (2 and 4); and between abattoirs (2 and 4) and eleven retailers, respectively. It shows, for instance, farm three ought to supply 800 livestock to abattoir one and 1200 livestock to abattoir four. Abattoir two ought to supply 850 packages of processed meats to retailer one and 210 packages of processed meats to retailer three.

Table 7. The result of Pareto solutions in terms of optimum quantity of product flow throughout the three-echelon meat supply chain.

\begin{tabular}{llllll}
\hline Facilities & Quantity & Facilities & Quantity & Facilities & Quantity \\
\hline $\mathrm{u}_{1,4}$ & 1200 & $\mathrm{v}_{2,1}$ & 850 & $\mathrm{v}_{2,11}$ & 700 \\
$\mathrm{u}_{3,1}$ & 800 & $\mathrm{v}_{2,3}$ & 210 & $\mathrm{v}_{4,6}$ & 850 \\
$\mathrm{u}_{3,4}$ & 1200 & $\mathrm{v}_{2,6}$ & 690 & $\mathrm{v}_{4,7}$ & 450 \\
$\mathrm{u}_{4,1}$ & 1000 & $\mathrm{v}_{2,5}$ & 290 & $\mathrm{v}_{4,9}$ & 110 \\
$\mathrm{u}_{4,2}$ & 290 & $\mathrm{v}_{2,10}$ & 100 & $\mathrm{v}_{4,2}$ & 350 \\
$\mathrm{u}_{4,4}$ & 100 & $\mathrm{v}_{2,8}$ & 160 & $\mathrm{v}_{4,4}$ & 220 \\
\hline
\end{tabular}

\section{Conclusions}


This paper presents a study in developing a multi-objective possibilistic programming model based on a three-echelon meat supply chain. The developed model comprises three objective functions aimed at (1) minimizing the total transportation cost (2) minimizing the required number of transportation vehicles and (3) minimizing the delivery time. Three methods are proposed in order to obtain the Pareto solutions and based on these to determine the optimal solution. Further, the developed model can be useful for decision makers to determine numbers of farms and abattoirs that need be established, and the quantity of livestock from farms to abattoirs and the quantity of meat products from abattoirs to retailers. In order to examine the applicability and effectiveness of the developed mathematical model that can be a useful tool for food supply chain designers, a case study was investigated based the collected data and the computational results were obtained using LINGO.

Future research may be considering multi-product multi-period production planning under different types of uncertainties and transportation means. Moreover, the environmental aspects can also be investigated as an objective function into the multi-objective model. This might be an important issue for large-size meat supply chains. In this way, the emissions due to the transport of all products throughout the meat supply chain should be determined.

\section{References}

Alonso-Ayuso, A., Escudero, LF., \& Ortuno, MT. 2007 On modeling planning under uncertainity in manufacturing, Stat Oper Res Trans., 31, 109-150.

Chan, F.T.S., Chung, S.H., \& Wadhwa, S. (2004). A hybrid genetic algorithm for production and distribution. Omega, 33, 345-355.

Chen, C.L., \& Lee, W.-C. (2004). Multi-objective optimization of multi-echelon supply chain networks, with uncertain product demands and prices. Computers and Chemical Engineering, 28, 1131-1144.

Deb, K. (2001). Multi-Objective Optimization using Evolutionary Algorithms. New York, John Wiley \& Sons.

Chankong, V., \& Haimes, Y. (1983). Multi-objective decision making theory and methodology. New York: Elsevier Science.

Ehrgott, M. (2005). Multicriteria Optimization. $2^{\text {nd }}$ ed., Springer, New York.

Miettinen, K. (1998). Nonlinear Multiobjective Optimization. Ed. $1^{\text {st }}$. Springer US: Springer Science+Business Media New York.

HMC, UK. (2010). http://www.halalhmc.org/ , Available: http://www.halalhmc.org/testdemo.htm . (accessed October 26, 2014).

Jiménez López, M., Arenas, M., Bilbao, A. \& Rodriguez, M.V. (2007). Linear programming with fuzzy parameters: An interactive method resolution. Eur. J. Oper. Res., 177, 1599-1609. 
Konak, A., Coit, C.W., \& Smith, A.E. (2006). Multi-objective optimization using genetic algorithms: a tutorial. Reliab. Eng. Syst. Saf, 91, 992-1007.

Meier, R.L., Williams, M.R., \& Singley, R.B. (2012). Supply Chain Management: Strategic Factors From The Buyers' Perspective. Journal of Industrial Technology, 20, $1-8$.

Mohammed, A. \& Wang, Q. (2015). Integrity of an RFID-enabled HMSC network. Proceedings of the Third International Conference on Digital Enterprise and Information Systems. China, pp. 79-86, 2015.

Niknamfar, A.H. (2015), "Multi-objective production-distribution planning based on vendor-managed inventory strategy in a supply chain", Industrial Management \& Data Systems, 115, 6, $1086-1112$.

Nozick, L., \& Turnquist, M. (2001). Inventory, transportation, service quality and the location of distribution centers. European Journal of Operations Research, 129, 362371.

Paksoy, P., Özceylan, E., \& Weber, G.-W. (2010). A Multi-Objective Mixed Integer Programming Model For Multi Echelon Supply Chain Network Design and Optimization. System Research and Information Technologies, 2, 519-854.

Ramesh, S., Kannan, S., \& Baskar, S. (2012). Application of modified NSGA-II algorithmto multi-objective reactive power planning. Appl. Soft Comput, 12, 741-753.

Revelle, C.S., \& Laporte, G. (1996). The plant location problem: New models and research prospects. Operations Research, 44, 864-874.

Sabri, B.H., \& Beamon, A. (2000). A multi-objective approach to simultaneous strategic and operational planning in supply chain design. Omega, 28, 581-598.

Schütz, P., Tomasgard, A., \& Ahmed, S. (2008). Supply chain design under uncertainty using sample average approximation and dual decomposition. European Journal of Operational Research, 199, 409-419.

Shankar, B.L., Basavarajappa, S., Jason, C.h., \& Chen, S. (2013). Location and allocation decisions for multi-echelon supply chain network - A multi-objective evolutionary approach. Expert Systems with Applications, 40, 551-562.

Simchi-Levi, D., Kaminsky, P., \& Simchi-Levi, D. (2001). Designing and managing the supply chain, New York, Irwin McGraw-Hill.

Sourirajan, K., Ozsen, L., \& Uzsoy, R. (2009). A genetic algorithm for a single product network design model with lead time and safety stock considerations. European Journal of Operational Research, 197, 599-608.

Snyder L (2006) Facility location under uncertainty: a review. IIE Trans 38(7):537-554.

Venkatesan, V., \& Kumanan, S. (2012). A multi-objective discrete particle swarm optimization algorithm for supply chain network design. International Journal of Logistics Systems and Management, 11, 375-406.

Listes, O. 2007. A generic stochastic model for supply-and-return network design, Comput. Oper. Res., 34, 417-442.

El-Sayed, M., Afia, N., \& El-Kharbotly, A. 2010. A stochastic model for forward_reverse logistics network design under risk, Comput. Ind. Eng., 58, 423-431.

Wang, H.F., Hsu, H.W. 2010. Resolution of an uncertain closed-loop logistics model: an application to fuzzy linear programs with risk analysis, J. Environ. Manage., 91, 214862. 
Qin, Z., Ji, X. 2010. Logistics network design for product recovery in fuzzy environment, Eur. J. Oper. Res. 202, 479-490.

Gholamiana, N., Mahdavia, I., Tavakkoli-Moghaddamb, R., \& Mahdavi-Amiric, N. 2015. Comprehensive fuzzy multi-objective multi-product multi-site aggregate production planning decisions in a supply chain under uncertainty, Applied soft computing, 37, 585-607.

Kannan, D., Khodaverdi, R., Olfat, 1., Jafarian, A., \& Diabat, A.I. 2013. Integrated fuzzy multi criteria decision making method and multi-objective programming approach for supplier selection and order allocation in a green supply chain, J. Clean. Prod., 47, 355367.

Aliev, R. A., Fazlollahi, B., Guirimov, B. G., \& Aliev, R.R. 2007. Fuzzy-genetic approach to aggregate production-distribution planning in supply chain management, Information Sciences, 177, 4241-4255.

Petrovic, D., Roy, R., \& Petrovic, R. (1998). Modelling and simula-tion of a supply chain in an uncertain environment, European Journal of Operational Research, 109(2), 299309.

Saha, A., Kar, S., Maiti, M. 2015. Multi-item fuzzy-stochastic supply chain models for long-term contracts with a profit sharing scheme, Applied Mathematical Modelling, 39, 2815-2828.

Wang, J. Shu, Y.F. 2007. A possibilistic decision model for new product supply chain design, European Journal of Operational Research, 177, 1044-1061.

Wang, Q., McIntosh, R., and Brain, B. 2010. "A new-generation automated warehousing capability”, International Journal of Computer Integrated Manufacturing, 23 (6): 565573.

Talaei, M., Farhang B., Pishvaee, M.S., Bozorgi-Amiri, A., \& Gholamnejad, S. 2015. A robust fuzzy optimization model for carbon-efficient closed-loop supply chain network design problem: A numerical illustration in electronics industry, Journal of Cleaner Production, 15, 959-6526.

Zarandi, M.H.F., Sisakht, A.H., \& Davari, S. 2011. Design of a Closed-loop Supply Chain (CLSC) Model Using an Interactive Fuzzy Goal Programming, The International Journal of Advanced Manufacturing Technology, 56, 809-821.

Sahar, V., Arijit B., \& Byrne, P.J. 2014. A case analysis of a sustainable food supply chain distribution system-A multi-objective approach, International Journal of Production Economics, 152, 71-87.

Robinson, D.R., \& Wilcox, S. 2008.The greening of supply chains. Supply Chain Manag. Rev., 12, 61-66.

Pagell, M., \& Wu, Z. 2009. Building a more complete theory of sustainable supply chain management using case studies of 10 exemplars. J. Supply Chain Manag. Glob. Rev. Purch. Supply, 45, 37-56.

Rong, A., Akkerman, R., \& Grunow, M.. 2011. An optimization approach for managing fresh food quality throughout the supply chain, Int. J. Prod. Econ., 131, 421-429. 